\title{
The Noetic Feeling of Confusion
}

\begin{abstract}
Feeling confused can sometimes lead us to give up on the task, frustrated. What is less emphasized is that confusion may also promote happy (epistemic) endings to our inquiries. It has recently been argued that confusion motivates effortful investigative behaviors which can help us acquire hard-to-get epistemic goods (DiLeo et al., 2019; D’Mello \& Graesser, 2012). While the motivational power of confusion and its benefits for learning has been uncovered in recent years, the exact nature of the phenomenon remains obscure. In this paper we attempt to shed light on the nature and epistemic value of an experience we are all familiar with: the experience of being confused at an object, a statement, etc. We first review the psychological literature on confusion, where it is most often considered to be an epistemic emotion. We then propose a refined account of confusion, by drawing on the literature on metacognitive or noetic feelings, both in psychology and in the philosophy of mind. In particular, we claim that confusion centrally involves the experience of the limits of one's cognitive capacities, because it results from a monitoring of our cognitive activities as we encounter a cognitive obstacle while processing a given content. Finally we show how our account may explain findings about the role of the experience of confusion in motivating deeper inquiry into complex problems and bringing about epistemic success in these cases.
\end{abstract}

Keywords: confusion; emotion; epistemic emotion; noetic feeling; metacognition; negative affect; learning. 


\section{Introduction}

Imagine you are given a mathematical problem. After a first look at it, you realize that you have no idea how to solve it or even how to start with it: the information just doesn't add up. You start frowning, you give it a closer look, but you remain stuck. What will you do next? Will you keep trying to make sense of the information you are presented with, or will you give up and move on to the next exercise?

The example above might be considered a typical instance of confusion. Dewey (1910, p. 12) claimed that: "The origin of thinking involves some perplexity, confusion, or doubt. Thinking is not a case of spontaneous combustion". Experiences like perplexity and confusion might be indispensable ingredients of our epistemic endeavors. Inquiry, the complex process which sometimes results in cognitive improvement, needs an initial spark. In spite of its bad reputation, we think that confusion can and often does act as one.

Even if most episodes of confusion are only accompanied by a mild feeling, this feeling is negatively valenced, and it can sometimes lead us to give up on the task, leaving us frustrated (D'Mello et al., 2014). This negative outcome of confusion has tended to overshadow another possible trajectory: one in which being confused results in a happy (epistemic) ending. Even if confusion can lead us to abandon a task when it is experienced with a high degree of intensity, it is a state which also motivates us to engage with the complex task we face. More specifically, it has recently been argued that confusion motivates effortful investigative behaviors which can participate in our ability to resolve complicated puzzles, and acquire hard-to-get epistemic goods as a result (DiLeo et al., 2019; D’Mello \& Graesser, 2012).

While the motivational power of confusion and its benefits for learning has been uncovered in recent years, the exact nature of the phenomenon remains somewhat obscure. This paper is an attempt to shed light on the nature and epistemic value of being confused in front of an object, an event, a statement, etc. In the first section we review the psychological literature on confusion where it is most often considered to be an emotion, and more precisely an epistemic emotion. Given that confusion centrally involves the experience of the limits of one's cognitive capacities in the face of a cognitive task, in section II we draw from the literature both in philosophy and in psychology on metacognitive or "noetic" feelings, to propose a refined account of mechanisms behind our experience of confusion. Noetic feelings are affective states which provide information regarding the course of our ongoing cognitive operations. In accordance with the "competence view" of noetic feelings (Dokic, 2012), we propose to view confusion as a feeling which results from a metacognitive monitoring of our cognitive operations, and which informs us on our cognitive competence at a task in the face of a cognitive obstacle. In section III we introduce recent empirical observations in educational psychology showing a correlation between the experience of confusion and tendencies to invest more cognitive resources and efforts in the search for a resolution. In section IV we show how our refined model of confusion may 
explain the role of confusion in bringing about these beneficial responses, thereby contributing to epistemic success in these cases.

\section{The emotion of confusion}

In their 2003 seminal paper on confusion, Rozin \& Cohen asked college students to identify, via target reports, the emotions that others expressed in the flow of spontaneous interaction (Rozin \& Cohen, 2003). To their surprise, the second most common descriptor reported by participants was confusion, a term that was virtually nonexistent in the affect or emotion literature. They further noted that confusion seemed to be endowed with many of the properties commonly attributed to emotions. Since then, a few authors in the field of educational psychology have taken interest in studying the impact of confusion on learning (e.g. D'Mello et al., 2014). Within this field, confusion has generally been conceptualized as an emotion particularly prominent in the context of learning and acquiring complex information (Arguel et al., 2017). How should we understand our phenomenon of interest if it is to be characterized as an emotion?

Emotions are affective mental states typically recognized to have a felt character (subjective feeling), to go along with some physiological changes, to have a positive or a negative valence (except for a few exceptions such as surprise), and to either result from or constitute an evaluation of an event or an object (Frijda, 1986; Lazarus, 1991; Scherer, 1999). The experience of being confused seems to carry all the features commonly attributed to emotions: when I feel confused, I experience a negative affect, I present some changes in my facial expression and bodily posture, and the confusion I feel is (or results from) an evaluation of the problem I am facing.

Emotions can further be categorized as belonging to various types, such as aesthetic emotions, moral emotions, etc. depending on the nature of the evaluative property they track. Moral emotions respond to "moral" values, like the just and the wicked and aesthetic emotions are thought to track "aesthetic" values like the sublime. Confusion has often been categorized as belonging to a class of emotions which track "epistemic" values and are thought to play a distinct role in guiding and regulating our epistemic activities.

In psychology, "epistemic emotions" are defined as "emotions that are caused by cognitive qualities of task information and the processing of that information" (Muis et al., 2015, p. 169). This means that epistemic emotions arise when one is facing information that one evaluates as new, incongruent, complex or different from one's previous knowledge. These properties (complex, new, incongruent, incoherent) are viewed as the typical evaluative properties which epistemic emotions track in objects (Pekrun \& Linnenbrink-Garcia, 2012). Pekrun (2021) recently proposed that epistemic emotions can be seen as generally instigated by appraisals of cognitive incongruity and a wish to resolve the incongruity. 
The most commonly referred to of such emotions is perhaps curiosity, but other affective states such as wonder, interest, surprise, feelings of certainty and confidence, are argued to fall into this category (Morton, 2009). Within the psychological literature, the most extensively studied epistemic emotions are surprise, curiosity, enjoyment, confusion, anxiety, frustration and boredom (Pekrun et al., 2017). Interestingly, this category of emotion was already mentioned by Darwin, who simply named them "intellectual emotions" (Darwin, 1872), thus highlighting how inextricable these emotions are from the cognitive processes they generate (Ellsworth, 2003).

Within the controversial class of epistemic emotions, further distinctions have been drawn. Some epistemic emotions are elicited when cognitive improvement is actually taking place, that is, when an epistemic gap has been filled, like feelings of understanding or eureka experiences. These affective states act as indicators that cognitive operations have been successful in producing an output or solution to the problem, and that inquiry can hence be closed. Other types of epistemic emotions arise in the context of potential cognitive improvement, like curiosity ; these can be called "exploratory affects" (Lauria, forthcoming). Finally, "emotions of darkness" - which include perplexity, confusion, doubt, and puzzlement - involve the experience of a cognitive obstacle; they signal a lack of cognitive improvement. Emotions of darkness, like exploratory affects, involve the appraisal of a gap in one's cognition, they can be distinguished on the basis of the evaluative component which concerns our coping potential, or the way we view events in light of our ability to act on them. In emotions of darkness such as confusion, or puzzlement, cognitive coping is appraised as low, and one experiences an obstacle, while this is not necessarily the case in exploratory affects.

Such a specification has been confirmed by other authors, who highlight the importance of cognitive coping in the emergence of confusion. Silvia (2010) notably highlights the impact of the novelty, complexity, and coping potential appraisals on the emergence of curiosity and confusion. An information is appraised as novel, complex, or unexpected, depending on the subject's existing knowledge and expectations for the task (Silvia, 2010; Turner \& Silvia, 2006). Different combinations of "epistemic" appraisals will give rise to different emotions: if information is appraised as new, complex, and comprehensible, subjects will experience interest (Silvia, 2005; Silvia et al., 2009). In contrast, if the information is appraised as not comprehensible, subjects will experience confusion (Silvia, 2010). Like curiosity, confusion involves appraising an object as new and complex; unlike curiosity, it involves appraising the event as hard to understand. In sum, our cognitive coping potential is appraised as low when we experience confusion (Muis et al., 2018).

As has become apparent in the distinctions drawn above, confusion involves the experience of the limits of our cognitive capacities. The fact that confusion provides information that regards one's own cognitive capacity or current epistemic position has been relatively unexplored in the literature. In the next section, we draw from the literature on metacognitive or 
"noetic" feelings to propose a model of confusion as a feeling which informs us on our own cognitive competence with regard to a given content or problem.

\section{The feeling of being confused}

Here we want to argue that, although we view confusion as an emotion, we believe the emotion literature has overlooked a critical dimension of the experience of confusion, namely the fact that the affective experience of confusion results from a tracking of one's own cognitive activities as they unfold to process a given content or resolve a certain problem. This dimension has however been better captured and modeled within the literature on metacognitive or "noetic" feelings.

Certain affective phenomena, such as the feeling of familiarity, the feeling of forgetting, or the tip-of-the-tongue feeling, are said to constitute a distinct class of affect, which seem to have this in common that they are directed at our cognitive states, and provide us with information on the unfolding of our cognitive activities. Noetic feelings are affective experiences directed at one's own epistemic states, events, and skills - their content is related to knowledge, ignorance, or uncertainty and they essentially tell the subject about the course of her own mental operations (de Sousa, 2008; Dokic, 2012). In psychology, they are traditionally referred to as "metacognitive feelings" (e.g. Efklides, 2006). It is commonly acknowledged that we often rely on such epistemic feelings in our ordinary reasoning, and that we also sometimes exploit them as premises in deliberation.

By contrast, epistemic emotions such as curiosity, interest, and surprise are not thought to primarily monitor the properties of our ongoing cognitive activities, but instead to monitor and signal properties of objects or situations that we encounter in the world (Meylan, 2014). They are thought to present these situations and objects to us as novel, incongruent, unexpected, complex, etc. Insofar as they produce these evaluations based on the subject's previous experiences and prior knowledge, emotions always have the subject's existing cognitive state as a reference point. In this sense it could be argued that the property of novelty that is attached to an object is always relational. However, emotions are not primarily conceived as directed at our internal states in the way that metacognitive or noetic feelings are thought to be.

In this paper we are not aiming to resolve the question of whether there is indeed a fundamental distinction between epistemic emotions and noetic feelings. Instead, we want to highlight the fact that the literature on noetic feelings, particularly in philosophy of mind and philosophy of cognitive science, offers a way to capture an important aspect of the experience of confusion, which seems to be absent from the emotion literature. This said, we believe that it is pos-

sible to adopt the model of confusion that we propose, while maintaining that confusion is an emotion. 
Epistemic feelings, as Koriat puts it (2000), are "cross-level states": they are conscious states which can be used as premises in reasoning just like any other piece of information, and are grounded on unconscious monitoring mechanisms. These feelings carry information (uncertainty, rightness, etc.) about the properties of our cognitive operations, but we do not have access to the event which caused the mechanism to launch this signal. Examples of such phenomena in the literature notably include: feelings of knowing/not knowing (Koriat, 1995, 2000), tip-of-thetongue experiences (Brown, 2000), feelings of competence (Bjork and Bjork 1992), feelings of familiarity (Whittlesea et al. 2001) and feelings of 'déjà vu' (Brown 2003). A notorious example is the feeling of knowing, which is a feeling one might experience while trying to retrieve a specific information from memory. The feeling is thought to signal that one does possess the information and should shortly be able to access that information consciously.

The nature of these states is, however, still a matter of ongoing debate. Particularly, it is still an open question whether these states imply metarepresentation or mind reading capacities, or whether they operate at a lower level and are experienced by non-human animals and young infants (Carruthers, 2008). Several authors have proposed that two levels of the metacognitive capacity can be distinguished (Koriat, 2000; Arango-Muñoz, 2011). Although these two levels can interact, they have different functions and require different capacities. The lower level of metacognition is thought to consist merely in a capacity to monitor and control cognitive operations. The capacity to monitor and control cognitive operations does not imply that one forms a second-order representation about those operations or their correlate mental states. This type of processes involve what Dokic (2012) calls "procedural metacognition", which consists in implicit monitoring and control of first-order processes. Procedural metacognition involves mechanisms deployed implicitly to monitor the quality of our cognitive processes in order to generate spontaneous actions.

Instead, the higher level of metacognition consists in the act of forming a second-order thought about a first-order one, and may be said to amount to no more than "turning our mindreading capacities upon ourselves" (Carruthers, 2009, p.8). It is the type of metacognition we engage in when we question, reassess, or evaluate our first-order thoughts in terms of reliability, accuracy, etc. This type of metacognition requires meta-representational capacities and, arguably, the possession of psychological concepts - such as perception, belief, intention, knowledge which the subject ascribes in order to interpret behavior. Such metacognitive judgement is hence composed of a proposition, a first-order attitude directed to it, and a second-order attitude directed at the first-order attitude and the original proposition (Proust, 2007; Arango-Muñoz, 2011). In functional terms, noetic feelings are thought to monitor cognitive processes and motivate epistemic decisions accordingly, either before the action is launched or after it has been performed. Applying this to confusion: when I feel confused about what a certain statement means, I am trying to understand the meaning of it; I am engaged in this cognitive activity, and my feeling of 
confusion arises to provide information about how my cognitive operations are proceeding for this task.

Now, what would the content of such a mental state be? If we were to try and translate it in ordinary language, it might be something like "I do not understand this". In a similar way, the feeling of knowing might be said to signal that: "I know this". Knowledge and understanding are epistemic goods which one seeks to achieve when performing cognitive tasks. While the feeling of knowing signals that such cognitive achievement has taken place and therefore that one now securely possesses the desired information, on this view the feeling of confusion signals that one has not reached the desired mental state; that cognitive operations have not yet delivered an output that qualifies as understanding. On this view, feeling confused implies that one possesses and deploys metarepresentational abilities, or the ability to represent one's own mental states, since it requires one to represent that one has not yet achieved the desired epistemic mental state of understanding. On this account, only creatures who possess the ability to represent their own mental states may experience confusion. Is there a less demanding way to conceive of the content of confusion?

One might think that because noetic feelings track epistemic states, their content can only be about those epistemic states, but there is another way to conceptualize the content of these feelings which does not require subjects to deploy metarepresentational abilities. According to a certain view of noetic feelings, noetic feelings are about one's own cognitive competence, in a way that is akin to feelings of physical competence (Dokic, 2012). Feelings of physical competence are affective experiences which are thought to help us gauge our competence at certain physical tasks. In other words, they function to help us foresee our chances of success at performing a given physical action. When standing above a puddle on the edge of the sidewalk, for instance, you might get a feeling with the content: "Yes, I can do this jump".

In a similar manner, Dokic (2012) proposes that noetic feelings signal one's cognitive competence at a task, helping us envisage our chances of success at resolving the problem or answering the question. For instance, when asked what the capital of Peru is, and before the answer comes to mind, one might get a feeling with the content: "Yes I can retrieve this information" or "No I cannot", which helps one foresee the result of one's performance and guides one's decision to persevere in one's cognitive efforts or give up trying to retrieve the information. According to this competence view of noetic feelings, the content of noetic feelings can accordingly be formulated in terms of: "I can/I cannot do this cognitive task". So conceived, the noetic feeling of confusion does not require the deployment of metarepresentational abilities. It is a feeling which helps us gauge our cognitive competence at a specific task, not by deploying metarepresentations concepts about our own cognitive states (like "I know this/ I don't know this"), but instead by gauging that our cognitive activity may or may not easily lead to the desired output ("I can/ I cannot do this"). 
Dokic argues more specifically that noetic feelings provide subjects with a type of modal knowledge on their cognitive competence. They yield information about what might easily happen, now or in the near future; particularly whether one's performance is or will be successful in possible worlds close to the actual world. For instance, according to this view a feeling of knowing is a feeling that one's performance is or will be successful in possible worlds close to the actual world. These worlds can be more or less close depending on the robustness of one's competence. Degrees of feelings can further be modeled in terms of the modal extent to which one's performance is successful, that is in terms of the closeness of the worlds in which one's performance is successful, given one's competence.

If we try to apply this model to the experience of feeling confused at a sentence or a scene, we might say the following: confusion is a feeling about one's cognitive competence in the sense that it appraises a cognitive obstacle as one which furthers our chances of succeeding at resolving a problem. It is a signal which arises as a result of facing a cognitive obstacle, and which functions to inform us that our cognitive competence has been challenged, and that our chances of success at the task are compromised. ${ }^{1}$ Additionally, it is a signal, as we will argue, which not only informs us that our performance may not easily lead to the desired output (given the cognitive obstacle detected), but which also conveniently triggers the recruitment of higherlevel, more demanding cognitive strategies in order to increase our chances of success. In short, confusion serves to alert us that a given cognitive task will not be so easily performed (thereby giving us information on our cognitive competence with respect to the task), and to launch the cognitive processes that will allow us to "think deeper" about the problem, thereby making it more likely for us to achieve understanding.

The feeling of confusion thus results from a monitoring of our own cognitive operations as they are unfolding, and is a way of apprehending how close or far we are from reaching an understanding of the given material. The satisfaction condition of confusion - what extinguishes it - is the achievement of understanding. Depending on the particular object that one's feeling of confusion is directed at (a statement, a mathematical problem, a novel object, etc.), achieving understanding can amount to such achievements as: disambiguating the information, identifying the source of the incoherence, resolving a contradiction, or finding a way in which the problem can be solved. When one achieves such an understanding and becomes conscious of it (be it through a feeling of understanding or a "aha" experience), confusion vanishes. What form must

\footnotetext{
${ }^{1}$ This is not to say that the feeling of confusion amounts or is identical with a lack of confidence. Experiences of confusion are likely to impact our level of confidence with regard to our ability to perform the task successfully. However, while levels of confidence amount to an epistemic stance, confusion is an emotional episode with distinct phenomenological features. In the same manner, other epistemic feelings, like feelings of knowing or not knowing, as defined by Koriat $(1995,2000)$ will inevitably impact one's level of confidence downstream regarding whether we will retrieve the information as needed. In this sense, such feelings may be exploited as indications and reasons to update one's level of confidence, i.e. one's graded epistemic stance on whether we will successfully complete the task or not.
} 
understanding take and what degree of understanding must be reached in order to extinguish one's feeling of confusion is likely to depend on the nature of the task itself, its relevance to one's goals, and on individual epistemic needs.

Based on the competence view of the feeling of confusion just sketched, can we shed some light on recent research concerning the positive impact of confusion on learning? In the next section we examine this literature and propose a novel perspective on the observed results.

\section{Feeling confused and learning}

On the view sketched above, confusion helps us gauge the feasibility of a cognitive task given one's competence. It helps explain why confusion triggers various behavioral and mental reactions such as a tendency to persevere and "think deeper". Indeed, if confusion was an experience of an object as literally incomprehensible, as some previous models seemed to suggest, it would just result in a motivation to terminate the ongoing inquiry. However, this is not systematically the case: confusion gives rise to a host of different reactions. According to our view, this is because confusion provides fine-grained information regarding our cognitive coping potential for the task we face, which we can then exploit in our practical reasoning about what to do next; in particular in deciding whether to persevere and invest the required amount of effort in the task, or give up on it.

In case one perseveres, success is not guaranteed, and if the costs (in time and energy) of inquiry come to overweight the predicted epistemic benefits, other emotions like frustration and boredom arise and might lead us to quit inquiry. When confusion is followed by frustration and boredom in this way, researchers speak of "hopeless confusion" (DiLeo et al., 2019). This is certainly a possible trajectory from confusion. At other times however, and perhaps more surprisingly, confusion can turn into "productive confusion" (DiLeo et al., 2019) and result in cognitive improvement. We now turn to such cases to examine what the exact role of confusion might be in bringing about epistemic success.

Empirical research from educational psychology has suggested that confusion may be driving epistemic success in complex learning tasks. Complex learning tasks require learners to generate inferences, diagnose and solve problems, generate explanations, demonstrate application and transfer acquired knowledge (Graesser et al., 2010). This form of deep learning can be contrasted with shallow learning activities (such as memorizing key phrases and facts) and simple forms of procedural learning. Importantly, complex learning tasks intrinsically convey cognitive disequilibrium, impasses, contradiction, etc. Confusion constitutes one way to respond to cognitive disequilibrium, and perhaps a particularly (epistemically) beneficial way.

In fact, D'Mello \& Graesser (2012) have shown that when subjects respond to cognitive disequilibrium with confusion, they seem to invest more effort in solving the problem and more 
cognitive resources in the search for a resolution. Confusion seems to promote a deeper kind of inquiry: a more thorough information processing which facilitates complex forms of learning. More work from D'Mello et al. (2014) shows that confusion may also be particularly beneficial when conceptual change is the aim of the task, where subjects need to overcome misconceptions before developing a more sophisticated and accurate representation of some material (Kennedy \& Lodge, 2016). It seems as though confusion helps subjects identify their erroneous approach to the material, but not only: confusion also seems to motivate a shift of approach, so that subjects experiencing confusion are more likely to look for and adopt alternative strategies to the problem.

Overall this literature suggests that experiencing confusion may be epistemically beneficial, because: 1) we work harder cognitively when we feel (mildly) confused, investing more cognitive effort in the epistemic task, and 2) we investigate differently, we seek alternative approaches and strategies. While these effects have been observed and documented, the exact role of the experience of confusion in bringing about these beneficial responses has not clearly been modeled. How can we account for the correlation between feeling confused and deploying the two aforementioned tendencies in inquiry? In the next and last section, we show how our model of confusion as sketched in section II may explain this correlation.

\section{Epistemic benefits of confusion}

We gather from the aforementioned studies that our faculty to feel confused might facilitate epistemic accomplishments that demand increased mental effort and shifts of strategy. This might, at first blush, appear puzzling. Experiences like curiosity and interest are generally the ones that are viewed as fit to motivate inquiry, since they appraise our cognitive coping potential as high (Silvia, 2010) ${ }^{2}$. In fact, one might think that confusion only results in deep inquiry when it is followed by exploratory kinds of affect which then provide the motivational power towards inquiry. Additionally, the studies just mentioned do not offer much explanation as to how - or in virtue of which of its properties - the feeling of confusion itself produces these effects, although they do attribute these effects to confusion and not to other emotions that might accompany or follow it.

Here is our idea of how confusion might function so that it sometimes produces behavioral tendencies conducive to epistemic success. Noetic feelings do not only monitor properties of our ongoing cognitive operations; they also have a control dimension. They trigger reactions to the events they monitor (Nelson and Narens, 1990). If confusion comprises a noetic feeling, it

\footnotetext{
2 While some authors refer to interest and curiosity equally (e.g. Silvia, 2010), recent work (Pekrun, 2019) suggest that both can be conceptualized as a trait and as a state. Here, we are only interested in curiosity as a state, that is, a short-lived emotional episode.
} 
then does not only monitor our cognitive operations, it is also partly constituted by a control dimension. According to this model, feeling confused not only provides us with information regarding our cognitive competence at a task and how easily we might be able to perform it, it also generates control behaviors accordingly. It sends us a signal with the content "I cannot do this task easily" and it automatically prepares us for putting in the effort that the task requires, given how far we are from successful performance.

Indeed, it is generally thought that the output of metacognitive monitoring activity also guides the regulation of control processes, and that learners exploit the subjective feelings produced by the monitoring activity to plan on how to invest their cognitive efforts. Hence, on the one hand, the metacognitive monitoring that leads to the subjective experience of confusion automatically triggers the recruitment of higher-level processes able to manage the not-so-easy task at hand. On the other hand, the subject can decide to consciously exploit the subjective feeling of confusion to plan her strategy around the learning task: either invest more effort and follow the inclination to "think deeper", or decide to drop or postpone her efforts at resolving the task. In other words, noetic feelings automatically exert a causal role on behavior, and subjects can also use the metaknowledge provided by them to regulate their information processing and cognitive investment (Dokic, 2012; Koriat, 2007).

Thus, even if we can decide to abandon inquiry when feeling confused (given other needs, desires, and pragmatic factors such as time pressure), the feeling of confusion encompasses a spontaneous inclination to launch the more demanding cognitive strategies required by the not-easily-feasible task. As has recently been argued, while effort is perceived as costly, humans as well as some non-human animals associate effort with reward, so that the investment of effort itself (and not only its products) is perceived to have a positive value (Inzlicht et al., 2018). This speaks in favor of the otherwise counter-intuitive idea that feeling confused about some information both makes the task appear more effortful, and inclines us to go forth with it.

If this picture of confusion is valid, in addition to allowing us to recognize that we are facing a cognitive obstacle, we have here suggested that feeling confused allows us to do so in a way which also triggers the recruitment of effortful cognitive strategies that are likely to help us overcome the obstacle and increase our chances of succeeding at the cognitive task. We suggest that future studies should assess how often confusion leads to either engagement or disengagement, as its impact will depend on the perceived size of the obstacle, the perceived value of removing it, and perceived coping potential (or control). 


\section{References}

Arguel, A., Lockyer, L., Lipp, O. V., Lodge, J. M., \& Kennedy, G. (2017). Inside Out: Detecting Learners' Confusion to Improve Interactive Digital Learning Environments. Journal of Educational Computing Research, 55(4), 526-551. https://doi.org/ 10.1177/0735633116674732

Bjork, R. A., \& Bjork, E. L. (1992). A new theory of disuse and an old theory of stimulus fluctuation. In A. F. Healy, S. M. Kosslyn, \& R. M. Shiffrin (Eds.), Essays in honor of William K. Estes. Hillsdale, NJ: Erlbaum.

Brown, S. R. (2000). Tip-of-the-tongue phenomena: An introductory phenomenological analysis. Consciousness and Cognition, 9(4), 516-537.

Brown, A. S. (2003). A review of the deja vu experience. Psychological bulletin, 129(3), 394.

Darwin, C. (1872). The Expression of the Emotions in Man and Animals. Cambridge University Press. https://www.cambridge.org/core/books/expression-of-the-emotions-inman-and-animals/0B7BDB02088055DAAB2FDB8DE304B47B

De Sousa, R. (2009). Epistemic feelings. Mind and Matter, 7(2), 139-161.

Di Leo, I., Muis, K. R., Singh, C. A., \& Psaradellis, C. (2019). Curiosity... Confusion? Frustration! The role and sequencing of emotions during mathematics problem solving. Contemporary Educational Psychology, 58, 121-137. https://doi.org/10.1016/ j.cedpsych.2019.03.001

D’Mello, S., \& Graesser, A. (2012). Dynamics of affective states during complex learning. Learning and Instruction, 22(2), 145-157. https://doi.org/10.1016/j.learninstruc. 2011.10.001

D’Mello, S., Lehman, B., Pekrun, R., \& Graesser, A. (2014). Confusion can be beneficial for learning. Learning and Instruction, 29, 153-170. https://doi.org/10.1016/j.learninstruc.2012.05.003

Dokic, J. (2012). Seeds of self-knowledge: noetic feelings and metacognition. Foundations of metacognition, 6, 302-321.

Ellsworth, P. C. (2003). Confusion, concentration, and other emotions of interest: Commentary on Rozin and Cohen (2003). Emotion, 3(1), 81-85. https://doi.org/10.1037/15283542.3.1.81

Frijda, N. H. (1986). The emotions. Cambridge University Press.

Graesser, A., Ozuru, Y., \& Sullins, J. (2010). What is a good question? In Bringing reading research to life. (Guilford Press., pp. 112-141). M. G. McKeown \& L. Kucan.

Kennedy, G., \& Lodge, J. M. (n.d.). All roads lead to Rome: Tracking students' affect as they overcome misconceptions. 11. 
Koriat, A. (1995). Dissociating knowing and the feeling of knowing: Further evidence for the accessibility model. Journal of Experimental Psychology: General, 124(3), 311.

Koriat, A. (2000). The feeling of knowing: Some metatheoretical implications for consciousness and control. Consciousness and cognition, 9(2), 149-171.

Koriat, A. (2007). Metacognition and consciousness. Cambridge University Press.

Lauria, F. (forthcoming). Interest and the Affective Springs of Inquiry - The "Chiaroscuro" Epistemic Emotion.

LeDoux, J. E., \& Hofmann, S. G. (2018). The subjective experience of emotion : A fearful view. Current Opinion in Behavioral Sciences, 19, 67-72. https://doi.org/10.1016/j.cobeha.2017.09.011

Lazarus, R. S. (1991). Emotion and adaptation (pp. xiii, 557). Oxford University Press.

Meylan, A. (2014). Epistemic emotions: a natural kind?. Philosophical inquiries, 2(1), 173190.

Morton, A. (2009, December 3). Epistemic Emotions. The Oxford Handbook of Philosophy of Emotion. https://doi.org/10.1093/oxfordhb/9780199235018.003.0018

Muis, K. R., Chevrier, M., \& Singh, C. A. (2018). The role of epistemic emotions in personal epistemology and self-regulated learning. Educational Psychologist, 1-20.

Muis, K. R., Pekrun, R., Sinatra, G. M., Azevedo, R., Trevors, G., Meier, E., \& Heddy, B. C. (2015). The curious case of climate change: Testing a theoretical model of epistemic beliefs, epistemic emotions, and complex learning. Learning and Instruction, 39, 168-183. https://doi.org/10.1016/j.learninstruc.2015.06.003

Nelson, T. O., \& Narens, L. (1990). Metamemory: A theoretical framework and new findings. In G. Bower (Ed.), The psychology of learning and motivation: Advances in research and theory (Vol 26, pp125-173). San Diego, CA: Academic Press.

Pekrun, R. (2019). The murky distinction between curiosity and interest: State of the art and future directions. Educational Psychology Review, 31, 905-914.

Pekrun, R., \& Linnenbrink-Garcia, L. (2012). Academic emotions and student engagement. In Handbook of research on student engagement (pp. 259-282). Springer. http:// link.springer.com/chapter/10.1007/978-1-4614-2018-7_12

Pekrun, R., Vogl, E., Muis, K. R., \& Sinatra, G. M. (2017). Measuring emotions during epistemic activities: The Epistemically-Related Emotion Scales. Cognition \& Emotion, 31(6), 1268-1276. https://doi.org/10.1080/02699931.2016.1204989

Rozin, P., \& Cohen, A. B. (2003). High frequency of facial expressions corresponding to confusion, concentration, and worry in an analysis of naturally occurring facial expressions of Americans. Emotion, 3(1), 68-75. https://doi.org/ $10.1037 / 1528-3542.3 .1 .68$ 
Scherer, K. R. (1999). Appraisal theory. In Handbook of cognition and emotion (pp. 637663). John Wiley \& Sons Ltd. https://doi.org/10.1002/0470013494.ch30

Silvia, P. J. (2005). What is interesting? Exploring the appraisal structure of interest. Emotion (Washington, D.C.), 5(1), 89-102. https://doi.org/10.1037/1528-3542.5.1.89

Silvia, P. J. (2010). Confusion and interest: The role of knowledge emotions in aesthetic experience. Psychology of Aesthetics, Creativity, and the Arts, 4(2), 75-80.

Silvia, P. J., Henson, R. A., \& Templin, J. L. (2009). Are the sources of interest the same for everyone? Using multilevel mixture models to explore individual differences in appraisal structures. Cognition \& Emotion, 23(7), 1389-1406. https://doi.org/ 10.1080/02699930902850528

Turner, S. A., \& Silvia, P. J. (2006). Must interesting things be pleasant? A test of competing appraisal structures. Emotion (Washington, D.C.), 6(4), 670-674. https://doi.org/ $\underline{10.1037 / 1528-3542.6 .4 .670}$

Whittlesea, B. W., \& Williams, L. D. (2001). The discrepancy-attribution hypothesis: I. The heuristic basis of feelings and familiarity. Journal of Experimental Psychology: Learning, Memory, and Cognition, 27(1), 3. 